\title{
ESTUDO TERMODINÂMICO DA INFLUÊNCIA DA MASSA DE ESCÓRIA E DE BANHO NO PROCESSO DE DESFOSFORAÇÃO EM PANELA*
}

Anna Paula Littig Berger ${ }^{1}$ Daniela Bahiense de Oliveira ${ }^{2}$ Cynara Christ Klippe/ ${ }^{3}$ Camila Santos Scope/ 4 José Roberto de Oliveira ${ }^{5}$

\section{Resumo}

A remoção do fosfóro através do processo de desfosforação do ferro-gusa tem sido cada vez mais relevante, devido a influência que exerce de forma prejudicial as propriedades do aço e devido a extensão do cenário de escassez de minérios com menor teores de fosfóro. Este trabalho tem como objetivo a avaliação termodinâmica de misturas à base de dolomita e finos de minério utilizadas no processo de desfosforação realizado em panela para o refino do ferro-gusa. Verificou-se uma tendência de maiores valores de eficiência para o processo de desfosforação quando aumenta-se a razão de massa de desfosforante e massa de gusa.

Palavras-chave: Pré-tratamento de ferro-gusa; Desfosforação; Termodinâmica.

\section{THERMODYNAMIC STUDY OF THE INFLUENCY OF SLAG AND BATH MASS IN THE PROCESS OF LADLE DEPHOSPHORIZATION}

\section{Abstract}

The removal of phosphorus through hot metal dephosphorization process has been increasingly important, since the injurious influences of phosphorus on the properties of steel and due the cenarium extension of scarce ore with lower phosphorus. This work aims to thermodynamic evaluation of mixtures based on dolomite and ore fines used in the process of ladle hot metal dephosphorization. Was possible to verify a tendency of major values for dephosphorization efficiency when increases the ratio between dephosphor mass and hot metal mass.

Keywords: Hot metal; Pre-treatment; Dephosphorization; Thermodynamic.

1 Engenheira Metalurgista e Mestranda em Engenharia Metalúrgica e de Materiais, Instituto Federal do Espírito Santo (IFES), Vitória, ES, Brasil.

2 Graduanda em Engenharia Metalúrgica e de Materiais, IFES, Vitória, ES, Brasil.

3 Engenheira Metalurgista e Mestranda em Engenharia Metalúrgica e de Materiais, IFES, Vitória, ES, Brasil.

4 Graduanda em Engenharia Metalúrgica, Instituto Federal do Espírito Santo, Vitória, ES, Brasil.

5 Prof. Dr., cursos de graduação e pós-graduação em Engenharia Metalúrgica e de Materiais, Instituto Federal do Espírito Santo (IFES), Vitória, ES, Brasil. 


\section{INTRODUÇÃO}

Inúmeras modificações e avanços tecnológicos foram e tem sido feitos a fim de aprimorar o processo de fabricação de aço para obtê-lo com maior limpidez, com velocidade de produção e o menor custo possível. ${ }^{1}$

A presença de fósforo no metal afeta adversamente algumas propriedades do aço como a tenacidade e a resistência. Além disso, a presença de fósforo causa a segregação intergranular durante o lingotamento contínuo e deteriora a ductilidade e resistência de produtos como tubos, aços criogênicos e placas de vasos de pressão².

A etapa de desfosforação de ferro-gusa pode ser realizada no carro torpedo, na panela de transferência de gusa ou até mesmo em um convertedor específico para este fim. Quando a DeP é realizada no convertedor, esta ocorre sem que haja uma redução do teor de carbono, e com duas etapas de vazamento de escória. Este procedimento é o que confere melhores resultado na DeP. ${ }^{1}$

Empresas ao redor do mundo implantaram e tem desenvolvido tecnologias de prétratamento de ferro-gusa. Entretanto, cada empresa adota um sistema de prétratamento adequado aos seus equipamentos existentes, layout e condições operacionais, onde as etapas de dessiliciação, dessulfuração e desfosforação são realizadas em equipamentos específicos para estes processos.

Algumas usinas japonesas já incorporaram etapas para redução do silício e do fósforo como operações de refino prévio do ferro-gusa, assim como a Siderúrgica Ibiraçu.

Com as exigências de teores de fósforo nos aços cada vez menores, e os teores de fósforo nos minérios de ferro cada vez maiores, os processos de desfosforação (DeP) de ferro-gusa ganham cada vez mais força.

Existem diferentes parâmetros da literatura para se avaliar termodinamicamente o processo de desfosforação de ferro-gusa, tais como o coeficiente de partição do fósforo (LP), a capacidade de fosfato $\left(\mathrm{CP}_{\mathrm{P}}\right)$ e a basicidade ótica $(\Lambda)$.

Por definição o coeficiente de partição do fósforo (Lp) é a razão no equilíbrio da porcentagem de fósforo presente na escória (\%Peq) e a porcentagem de fósforo presente no metal [\%Peq], conforme a equação 1.

$$
\mathrm{Lp}=\frac{\left(\% \mathrm{P}_{\text {eq }}\right)}{\left[\% \mathrm{P}_{\text {eq }}\right]}(1)
$$

Entretanto a reação de desfosforação ocorre na interface metal e escória e não ocorre no equilíbrio, dificultando a real medição desses valores. Por isso vários autores estudaram e definiram empiricamente equações que pudessem medir os valores do coeficiente de partição do fósforo, que podem ser visualizados na Tabela 1.

Tabela 1. Modelos termodinâmicos da literatura utilizados.

\begin{tabular}{|c|c|}
\hline Autor & Modelo \\
\hline Young & $\begin{array}{c}\log C p=-18,184+35,84 \cdot \Lambda-22,35 \cdot \Lambda^{2}+\frac{22 \cdot 930 \cdot \Lambda}{T}-0,06257 \cdot \% \mathrm{FeO} \\
-0,04256 . \% \mathrm{MnO}+0,359 . \% P_{2} O_{5}^{0,3}\end{array}$ \\
\hline Healy & $\log \frac{(\% P)}{[\% P]}=\frac{22.350}{T}+0,08 \cdot(\% C a O)+2,5 \cdot \log \left(\% F e_{\text {total }}\right)-16$ \\
\hline $\begin{array}{l}\text { Suito e } \\
\text { Inoue }\end{array}$ & $\log \frac{(\% P)}{[\% P]}=0,072[(\% C a O)+0,3 \cdot(\% M g O)]+2,5 \cdot \log \left(\% F e_{\text {total }}\right)+\frac{11 \cdot 570}{T}-10,52$ \\
\hline Turkdogan & $\log \left(\gamma_{P_{2} O_{5}}\right)=-1,12 \cdot\left(22 \cdot N_{C a O}+15 \cdot N_{M g O}+13 \cdot N_{M n O}+12 \cdot N_{F e O}-2 \cdot N_{S i O 2}\right)-\frac{42000}{T}+23,58$ \\
\hline
\end{tabular}


A remoção do fósforo do metal e a consequente fixação do óxido formado na escória dependem da atividade do $\mathrm{P}_{2} \mathrm{O}_{5}$ na escória, sendo esta em função da composição química e temperatura. Quanto maior a concentração de $\mathrm{P}_{2} \mathrm{O}_{5}$ na escória, maior será a concentração de fósforo no metal, conforme equação 2. Sendo $\mathrm{N}_{\mathrm{P} 205}$ a fração molar do composto.

A concentração de $\mathrm{P}_{2} \mathrm{O}_{5}$ na escória está diretamente relacionada a sua atividade na mesma, conforme é mostrado a seguir.

Turkdogan (1996) descreve a variação do coeficiente de atividade do $\mathrm{P}_{2} \mathrm{O}_{5}$ com a temperatura e composição da escória em uma relação empírica, mostrada na Tabela 1.

$$
a_{P_{2} o_{5}}=N_{P_{2} o_{5}} \cdot \gamma_{P_{2} o_{5}}
$$

A basicidade de cada óxido é calculada em função da eletronegatividade de Pauling e a basicidade teórica da escória é dada pela média ponderada das contribuições individuais, através da fração molar, conforme Equação 3 e Equação 4:

$$
\begin{gathered}
\Lambda_{i}=\frac{0,74}{(X-0,26)} \\
\Lambda_{e s c}=\sum \Lambda_{\mathbf{i}} N
\end{gathered}
$$

Onde $X$ é a eletronegatividade de Pauling, $\mathrm{N}$ fração molar do óxido ou fluoreto na escória, $\Lambda_{i}$ basicidade de cada óxido e $\Lambda_{e s c}$ basicidade da escória.

\section{MATERIAIS E MÉTODOS}

Para a realização do presente trabalho foram realizadas as seguintes etapas:

- Caracterização dos materiais usados nas corridas preliminares da Siderúrgica de Ibiraçu;

- Composição da mistura;

- Realização dos cálculos termodinâmicos;

\subsection{Caracterização dos Materiais}

Como base para o início dos estudos e aplicação dos conceitos técnicos foram feitas corridas com o procedimento padrão da Siderúrgica de Ibiraçu.

Ao término da redução do minério de ferro no alto forno, o ferro-gusa é vazado do para panela, onde será realizado o processo de desfosforação. Nesta etapa realizase uma primeira separação da escória do ferro-gusa.

Após o canal de corrida o ferro-gusa é vazado na panela de tratamento. No início do vazamento começa a injeção de nitrogênio pela parte inferior da panela. Quando o ferro-gusa cobre todo o fundo da panela, é iniciada a adição de mistura desfosforante. Essa mistura desfosforante é composta de uma relação mássica de $1: 1$ de dolomita e finos de minério de ferro. 
Tabela 2. Massa de desfosforante e massa de gusa utilizadas.

\begin{tabular}{ccc}
\hline Corrida & $\begin{array}{c}\text { Massa } \\
\text { desfosforante } \\
(\mathrm{kg})\end{array}$ & $\begin{array}{c}\text { Massa } \\
\text { de gusa } \\
(\mathrm{kg})\end{array}$ \\
\hline M1 & 1250 & 19280 \\
\hline M2 & 1250 & 19820 \\
\hline M3 & 1000 & 16950 \\
\hline M4 & 1000 & 23440 \\
\hline M5 & 750 & 19650 \\
\hline M6 & 750 & 23470 \\
\hline M7 & 1000 & 20490 \\
\hline M8 & 500 & 24320 \\
\hline M9 & 750 & 22110 \\
\hline M10 & 750 & 22040 \\
\hline M11 & 500 & 21860 \\
\hline M12 & 750 & 19730 \\
\hline
\end{tabular}

Após o término do carregamento do ferro-gusa na panela, inicia-se o sopro de oxigênio. Posteriormente ao término do sopro de oxigênio, a lança é retirada, e em seguida uma amostra do ferro-gusa é coletada para análise química.

Após iniciado o basculamento da panela para o vazamento do ferro-gusa tratado na máquina de moldar, uma amostra do ferro-gusa é coletada para análise química, e durante o vazamento é retirado à escória. A temperatura do processo se manteve em torno de $1370^{\circ} \mathrm{C}$.

Após a verificação dos procedimentos executados para o pré-tratamento da Siderúrgica de lbiraçu, foram coletadas amostras de 12 corridas, com as análises dos teores de $\mathrm{Si}, \mathrm{Mn}, \mathrm{P}, \mathrm{S}$ e $\mathrm{C}$ antes do pré tratamento na panela e após. Estes resultados estão apresentados na Tabela 3.

Tabela 3. Análise das corridas preliminares antes e após do tratamento

\begin{tabular}{|c|c|c|c|c|c|c|c|c|c|c|}
\hline \multirow{2}{*}{ Corrida } & \multicolumn{2}{|c|}{$\% S i$} & \multicolumn{2}{|c|}{$\% \mathrm{Mn}$} & \multicolumn{2}{|c|}{$\% P$} & \multicolumn{2}{|c|}{$\% S$} & \multicolumn{2}{|c|}{$\% \mathrm{C}$} \\
\hline & inicial & final & inicial & final & inicial & final & inicial & final & inicial & final \\
\hline M1 & 0,22 & 0,05 & 0,066 & 0,018 & 0,076 & 0,023 & 0,019 & 0,008 & 4,37 & 4,31 \\
\hline M2 & 0,2 & 0,07 & 0,06 & 0,026 & 0,074 & 0,027 & 0,014 & 0,008 & 4,35 & 4,39 \\
\hline M3 & 0,18 & 0,06 & 0,064 & 0,013 & 0,077 & 0,027 & 0,017 & 0,01 & 4,38 & 4,45 \\
\hline M4 & 0,14 & 0,07 & 0,053 & 0,013 & 0,073 & 0,024 & 0,019 & 0,008 & 4,32 & 4,38 \\
\hline M5 & 0,16 & 0,05 & 0,06 & 0,013 & 0,07 & 0,027 & 0,022 & 0,01 & 4,39 & 4,35 \\
\hline M6 & 0,1 & 0,06 & 0,065 & 0,009 & 0,076 & 0,03 & 0,017 & 0,008 & 4,36 & 4,49 \\
\hline M7 & 0,08 & 0,08 & 0,048 & 0,009 & 0,078 & 0,024 & 0,014 & 0,006 & 4,3 & 4,37 \\
\hline M8 & 0,15 & 0,08 & 0,055 & 0,026 & 0,075 & 0,03 & 0,017 & 0,006 & 4,38 & 4,2 \\
\hline M9 & 0,16 & 0,05 & 0,05 & 0,026 & 0,072 & 0,034 & 0,014 & 0,01 & 4,34 & 4,25 \\
\hline M10 & 0,23 & 0,06 & 0,066 & 0,018 & 0,071 & 0,032 & 0,01 & 0,008 & 4,38 & 4,39 \\
\hline M11 & 0,2 & 0,08 & 0,062 & 0,031 & 0,078 & 0,046 & 0,017 & 0,01 & 4,35 & 4,42 \\
\hline M12 & 0,22 & 0,08 & 0,066 & 0,031 & 0,074 & 0,038 & 0,01 & 0,006 & 4,34 & 4,4 \\
\hline
\end{tabular}




\subsection{Caracterização das Misturas}

No presente trabalho como a mistura usada foi na proporção de 1:1 entre a dolomita e os finos de minério, foi criada a Tabela 4 que mostra a composição da mistura, quando usado dolomita já no banho, ou seja, considerando as calcinações.

Tabela 4. Composição da mistura no banho

\begin{tabular}{cc} 
COMPONENTE & \% Componente no banho \\
\hline $\mathrm{FeO}$ & $65,98 \%$ \\
$\mathrm{CaO}$ & $19.99 \%$ \\
$\mathrm{MgO}$ & $8,27 \%$ \\
$\mathrm{SiO}_{2}$ & $1.89 \%$ \\
$\mathrm{Al}_{2} \mathrm{O}_{3}$ & $0,48 \%$ \\
$\mathrm{P}$ & $0,007 \%$ \\
$\mathrm{Mn}$ & $0,015 \%$ \\
$\mathrm{TiO}_{2}$ & $0,04 \%$ \\
$\mathrm{PPC}$ & $0,045 \%$ \\
$\mathrm{H}_{2} \mathrm{O}$ & $1,22 \%$ \\
$\mathrm{Outros}$ & $2,04 \%$ \\
\hline
\end{tabular}

Os resultados apresentados na Tabela 4, se mostram propícios ao processo de desfosforação apresentado pela Equação 5, devido aos teores de $\mathrm{FeO}$ e $\mathrm{CaO}$ aferidos.

$$
2 \underline{P}+5(\mathrm{FeO})+3(\mathrm{CaO})=\left(3 \mathrm{CaO} \cdot \mathrm{P}_{2} \mathrm{O}_{5}\right)_{(s)}+5 \underline{\mathrm{Fe}}
$$

Foi utilizado o software Factsage para determinar a quantidade de líquido e fases sólidas formadas nas misturas propostas no presente trabalho.

Foram utilizados os bancos de dados FactPS e FtOxid disponíveis nesse software. O primeiro atua na determinação dos compostos sólidos formados e o segundo atua na avaliação dos compostos formados na fase líquida da mistura/escória. Foram usados Pure Solids e Slag? do sub-banco de dados existente.

\section{RESULTADOS E DISCUSSÃO}

\subsection{Avaliação da eficiência da desfosforação através das misturas iniciais}

Para uma verificação dos resultados apresentados na Tabela 3 foi criada a Figura 1, a qual mostra para cada um dos parâmetros analisados ( $P$, Si, $S$ e $M n$ ), seu comportamento em relação ao tratamento em panela.

As Figuras 1 e 2 apresentam os valores da fração mássica inicial e final dos elementos analisados. Para se obter estes valores foi feito uma média aritmética das frações dos componentes para cada grupo de massa de desfosforante utilizada $(500,750,1000$ e $1250 \mathrm{~kg})$, e foi desconsiderada a massa de gusa. 

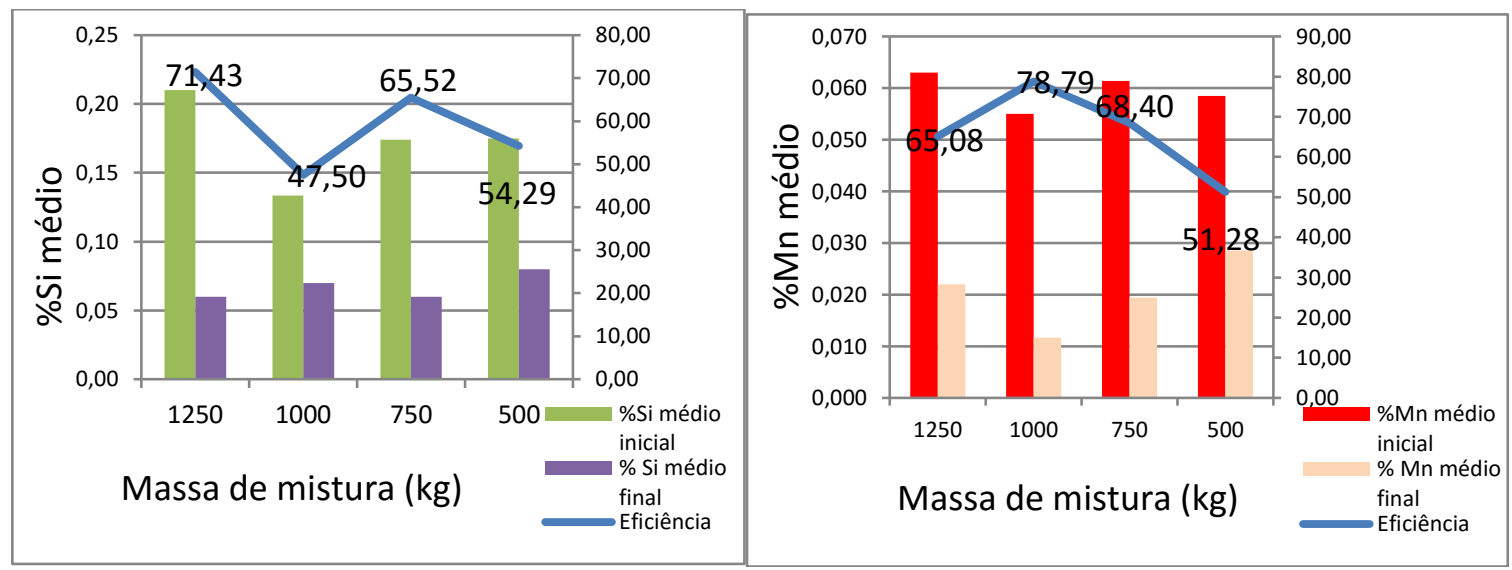

Figura 1. Redução média dos teores de (a) Si e (b) Mn em relação a massa de desfosforante aplicada.
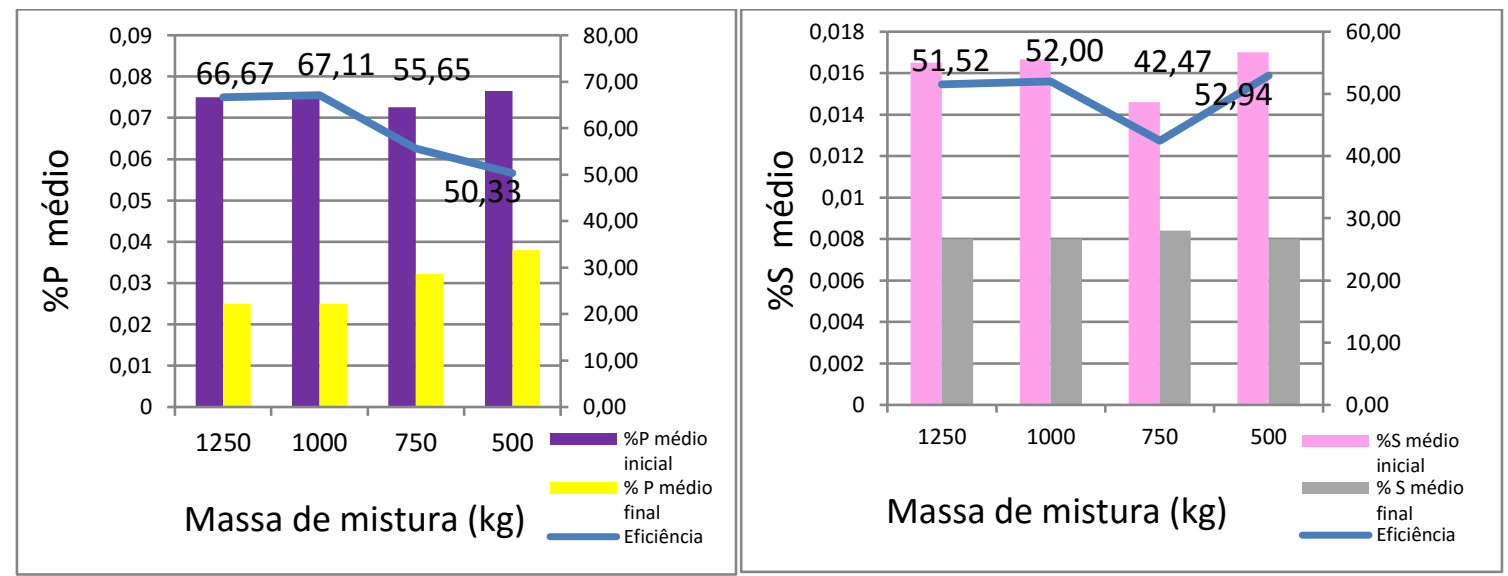

Figura 2. Redução média dos teores de (a) P e (b) S em relação a massa de desfosforante aplicada.

Pode-se observar pelas Figuras 1 e 2 que as massas de mistura de $1000 \mathrm{~kg}$ e 1250 $\mathrm{kg}$ apresentaram bons resultados de redução média dos teores de $\mathrm{Si}, \mathrm{Mn}, \mathrm{P}$ e S.

Em análise preliminar destes dados, pode-se concluir inicialmente que a massa ideal de desfosforante estaria entre 1000 e $1250 \mathrm{~kg}$, e para melhor análise foi criada a Tabela 5. 
Tabela 5. Dados das corridas

\begin{tabular}{ccccc}
\hline Corrida & $\begin{array}{c}\text { Desfosforante } \\
\mathbf{( k g )}\end{array}$ & $\begin{array}{c}\text { Gusa } \\
\mathbf{( k g )}\end{array}$ & $\begin{array}{c}\text { Massa de } \\
\text { DeP/Massa } \\
\text { de gusa } \\
\text { (kg/ton) }\end{array}$ & $\eta \%$ \\
\hline $\mathbf{8}$ & 500 & 24.320 & 20,56 & 60 \\
\hline $\mathbf{1 1}$ & 500 & 21.860 & 22,87 & 41,03 \\
\hline $\mathbf{1 2}$ & 750 & 19.730 & 25,34 & 51,35 \\
\hline $\mathbf{6}$ & 750 & 23.470 & 31,96 & 60,53 \\
\hline $\mathbf{9}$ & 750 & 22.110 & 33,92 & 52,78 \\
\hline $\mathbf{1 0}$ & 750 & 22.040 & 34,03 & 54,93 \\
\hline $\mathbf{5}$ & 750 & 19.650 & 38,17 & 61,43 \\
\hline $\mathbf{4}$ & 1000 & 23.440 & 42,66 & 67,12 \\
\hline $\mathbf{7}$ & 1000 & 20.490 & 48,8 & 69,23 \\
\hline $\mathbf{3}$ & 1000 & 16.950 & 59.00 & 64,94 \\
\hline $\mathbf{2}$ & 1250 & 19.820 & 63,07 & 63,51 \\
\hline $\mathbf{1}$ & 1250 & 19.280 & 64,83 & 69,74 \\
\hline
\end{tabular}

A Figura 3 apresenta o comportamento dos teores de $\mathrm{P}, \mathrm{Si}, \mathrm{S}$ e $\mathrm{MN}$ no metal em relação a massa de desfosforante por tonelada de gusa aplicado na corrida.

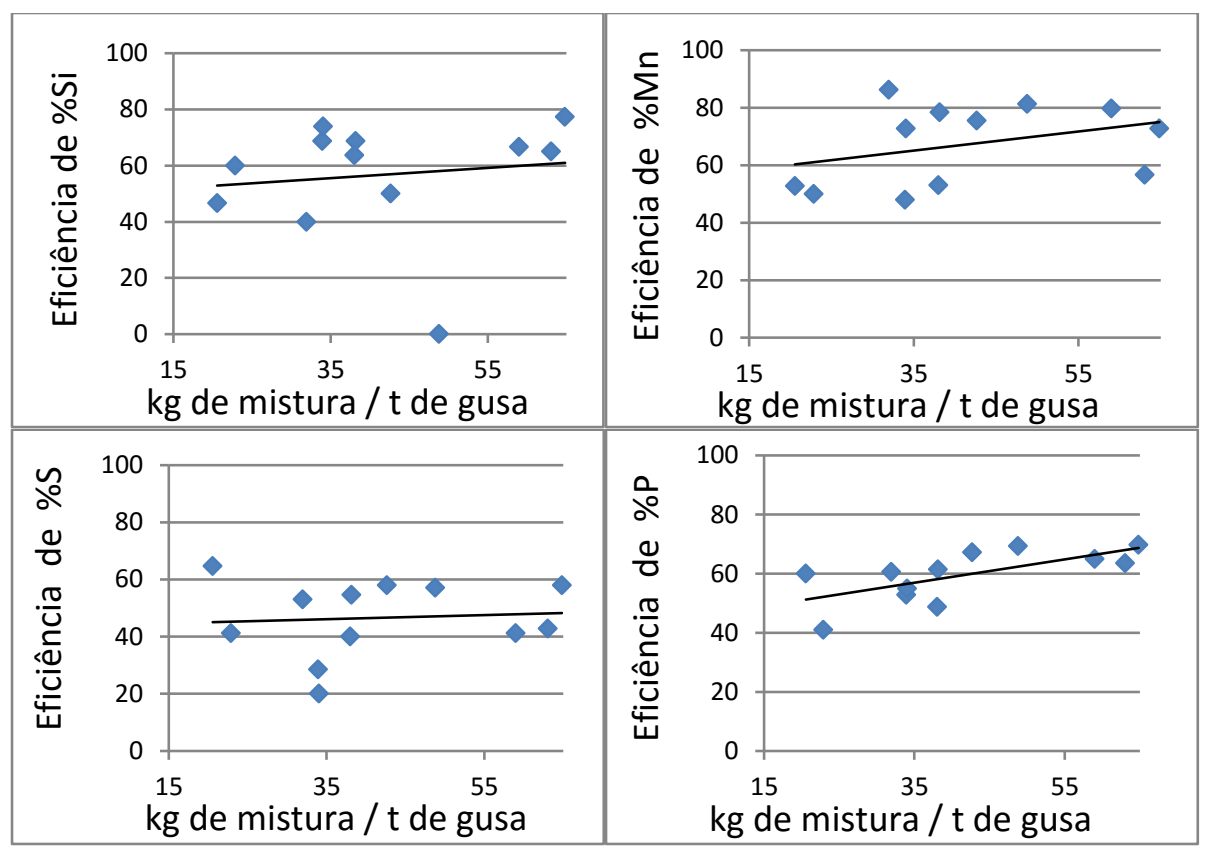

Figura 3. $\mathrm{P}, \mathrm{Si}, \mathrm{S}$ e $\mathrm{MN}$ no metal em relação à massa de desfosforante por tonelada de gusa para as corridas.

Observa-se que na Figura 3 o comportamento do teor de P, Si e Mn final no metal em relação à massa de desfosforante por tonelada de gusa aplicado na corrida, na qual verifica-se uma tendência de maior eficiência quando aumentamos a massa de desfosforante em relação à massa de gusa.

Para o S não se verifica esta tendência tão acentuada de maior ou menor eficiência quando aumentamos a massa de desfosforante em relação à massa de gusa, o que pode ser resultado da falta de controle da temperatura em cada corrida. 


\subsection{Avaliação termodinâmica com modelos literários}

A análise termodinâmica da desfosforação do ferro-gusa foi feita através dos modelos apresentados na Introdução, sendo a basicidade ótica, a capacidade de fosfato e o coeficiente de partição do fósforo. A capacidade de fosfato foi calculada a partir do modelo de Young (1991), o coeficiente de partição do fósforo foi calculado a partir dos modelos de Healy (1970) e Suito e Inoue (2006), e o coeficiente de atividade do $\mathrm{P}_{2} \mathrm{O}_{5}$ pelo modelo de Turkdogan (2000).

Tabela 6. Condições termodinâmicas do processo de desfosforação

\begin{tabular}{ccccc}
$\Lambda$ & $\begin{array}{c}\log (\mathrm{CP}) \\
\text { Young }\end{array}$ & $\begin{array}{c}\text { Log (LP) } \\
\text { Healy }\end{array}$ & $\begin{array}{c}\text { Log (LP) } \\
\text { Suito }\end{array}$ & у P2O5 \\
\hline 0,663 & 0,873 & 3,477 & 2,415 & $7,18 \mathrm{E}-19$ \\
\hline
\end{tabular}

Devido ao fato de que as misturas possuem a mesma composição, os valores dos parâmetros acima calculados são iguais para M1 a M12 independente da massa da mistura desfosforante, uma vez que esses parâmetros levam em consideração fração molar ou porcentagem dos componentes da mistura.

Contudo através da literatura observa-se que esses parâmetros não são precisos para prever a eficiência do processo de desfosforação uma vez que se tratam do equilíbrio termodinâmico. E a partir dos dados apresentados na Tabela 6 , não é possível relacionar diretamente a eficiência do processo de desfosforação com o aumento da basicidade ótica, a diminuição do coeficiente de atividade do $\mathrm{P}_{2} \mathrm{O}_{5}, 0$ aumento da capacidade de fosfato ou ainda do coeficiente de partição de fósforo da escória final.

De acordo com a literatura, quanto maior o coeficiente de partição, maior a eficiência do processo. Os valores encontrados não se mostram bons para melhor eficiência do processo de desfosforação quando comparados com a literatura. [1]

A equação 2 mostra que quanto menor o coeficiente de atividade de $\mathrm{P}_{2} \mathrm{O}_{5}$, menor será sua atividade e, assim, menor será a concentração de fósforo no metal. Da literatura, pode-se observar então que o valor obtido de coeficiente de $\mathrm{P}_{2} \mathrm{O}_{5}$ para as misturas é um bom valor. [1]

\section{CONCLUSÃO}

Considerando os objetivos propostos neste trabalho em paralelo aos resultados obtidos, pode-se chegar as seguintes conclusões:

- Verificou-se uma tendência de maior eficiência de desfosforação quando há um aumento da razão de massa de desfosforante e massa de gusa;

- Os parâmetros termodinâmicos calculados, coeficiente de partição do fósforo (LP), capacidade de fosfato $\left(\mathrm{C}_{\mathrm{P}}\right)$ e basicidade ótica $(\Lambda)$ não são precisos para prever a eficiência do processo de desfosforação uma vez que se tratam do equilíbrio termodinâmico;

- A massa ideal de desfosforante para o processo analisado estaria entre $1000 \mathrm{~kg} \mathrm{e}$ $1250 \mathrm{~kg}$ uma vez que foi observado melhores valores de redução do teor de $P$.

\section{Agradecimentos}

Ao CNPQ pela bolsa de iniciação científica do Edital PRPPG 05/2015. 


\section{REFERÊNCIAS}

1 BROSEGHINI, F.C. Utilização da termodinâmica computacional no estudo da desfosforação de ferro-gusa por escórias do Sistema CaO-FeO. Dissertação de mestrado. Instituto Federal Espírito Santo - IFES, 2015.

2 MUKHERJEE, T.; CHATTERJEE, A. Production of low phosphorus steels from high phosphorus Indian hot metal: Experience at Tata Steel. Bulletin of Material Science, $\mathrm{n}$. 6, v. 19, p. 893-903, 1996.

3 CAMPOS, V. F. Curso sobre Pré-refino de Ferro-Gusa. 1. ed. Belo Horizonte: Universidade Federal de Minas Gerais, 1984. 31p.

4 TURKDOGAN, E.T. Fundamentals of Steelmaking. 1. ed. London: The Institute of Materials, 1996. 331p.

5 HEALY, G. A New Look at Phosphorus Distribution. Journal of the Iron and Steel Institute, n. 6, v. 208, p. 664-668, 1970.

6 WEI, P.; SANO, M.; HIRASAWA, M.; MORI, K. Kinects of Phosphorus Transfer between Iron Oxide Containing Slag and Molten Iron of High Carbon Concentration under Ar-O2 Atmosphere. ISIJ International, n. 4, v. 33, p. 479-487, 1993.

7 HINO, Y.; KIKUCHI, N.; NABESHINA, S.; TAKEUCHI, S. Effect of iron oxide feeding rate and hot metal temperature on dephosphorization rate in torpedo car. ISIJ International, 827-834, 2005.

8 SAMPAIO, R. A. Utilização dos softwares de termodinâmica computacional no estudo das etapas de pré-tratamento do ferro-gusa. Dissertação de Mestrado. Insituto Federal Espírito Santo - IFES, 2014. 